\title{
Are age-related changes in cognitive function driven by age-related changes in sensory processing?
}

\author{
Larry E. Humes • Thomas A. Busey • James Craig • \\ Diane Kewley-Port \\ Published online: 20 December 2012 \\ (C) The Author(s) 2012. This article is published with open access at Springerlink.com
}

\begin{abstract}
Although there has been keen interest in the association among measures of sensory function and cognitive function for many years, in general, measures of sensory function have been confined to one or two senses and measures of threshold sensitivity (acuity). In this study, rigorous psychophysical measures of threshold sensitivity, temporal gap detection, temporal order identification, and temporal masking have been obtained, in hearing, vision, and touch. In addition, all subjects completed 15 subtests of the Wechsler Adult Intelligence Scale, 3rd edition (WAISIII). Data were obtained from 245 adults (18-87 years old) for the WAIS-III and for 40 measures of threshold sensitivity and temporal processing. The focus in this report is on individual differences in performance for the entire data set. Principal-components (PC) factor analysis reduced the 40 psychophysical measures to eight correlated factors, which were reduced further to a single global sensory processing factor. Similarly, PC factor analysis of the 15 WAIS-III scores resulted in three correlated factors that were further reduced to a single global cognitive function factor. Age, global sensory processing, and global cognitive function were all moderately and significantly correlated with one another. However, paired partial correlations, controlling for the third of these three measures, revealed that the moderate correlation between age and global cognitive function went to zero when global sensory processing was controlled for; the other two partial correlations remained intact. Structural models confirmed this result. These analyses suggest that the long-standing observation of age-related changes in
\end{abstract}

L. E. Humes $(\bowtie) \cdot$ D. Kewley-Port

Department of Speech \& Hearing Sciences, Indiana University,

Bloomington, IN 47405-7002, USA

e-mail: humes@indiana.edu

T. A. Busey $\cdot$ J. Craig

Department of Psychological \& Brain Sciences,

Indiana University,

Bloomington, IN 47405, USA cognitive function may be mediated by age-related changes in global sensory processing.

Keywords Aging · Cognition · Threshold · Temporal order · Temporal masking

Sensory function declines with advancing age, especially for hearing and vision (for reviews, see, e.g., Fozard, 1990; Schacht \& Hawkins, 2005). Likewise, there has been a long awareness of cognitive deficits with advancing age in otherwise healthy adults (e.g., Salthouse, 2010). More recently, over the past quarter century or so, researchers have turned their attention to the possible association of these two agerelated declines (e.g., Lindenberger \& Baltes, 1994; Schneider \& Pichora-Fuller, 2000). Schneider and PichoraFuller reviewed several of the competing theories about the association between sensory and cognitive decline and labeled them as follows: (1) the sensory deprivation hypothesis, (2) the information degradation hypothesis, (3) the cognitive-load-on-perception hypothesis, and (4) the common-cause hypothesis. Briefly, the sensory deprivation hypothesis and the information degradation hypothesis both suggest that cognitive decline is preceded by sensory decline, with the difference between these two hypotheses being in the time required for the sensory decline to have an impact (long vs. immediate or chronic vs. acute for the deprivation and degradation hypotheses, respectively). The cognitive-load-on perception hypothesis, unlike the two preceding hypotheses, suggests that age-related declines in cognition drive or precede measured changes in sensory processing. Finally, the common-cause hypothesis suggests that there is a common underlying factor that concurrently has a negative impact on sensory processing and cognitive function with advancing age.

Although there have been some exceptions, at least for measures of acuity in more than two senses (e.g., Stevens, Cruz, Marks, \& Lakatos, 1998), for the most part, studies of 
the association between sensory and cognitive function across the adult lifespan have involved (1) hearing, vision, or both; and (2) simple measures of sensory processing for each sense, such as Snellen charts for visual acuity or puretone audiometry for hearing acuity. Regarding the use of simple measures of sensory processing in most prior studies, there are several potential problems with this approach. First, the acuity measures used in each sense were not always criterion-free measures (Green \& Swets, 1966). There is some evidence that older adults employ a more conservative criterion when responding during psychophysical tasks (e.g., Clark \& Mehl, 1971; Gatehouse \& Davis, 1992; Gordon-Salant, 1986; Potash \& Jones, 1977; Ratcliff, Thapar, \& McKoon, 2007; Rees \& Botwinick, 1980), although this certainly has not been a universal finding (e.g., Marshall, 1991; Watson, Turpenoff, Kelly, \& Botwinick, 1979). If such an age-related response bias is present, however, this alone may result in common or shared variance across measures. Second, a hallmark of various psychophysical and cognitive measures obtained from older adults is that the measures tend to show much more within-subjects measurement variability than do comparable measures from young adults. In fact, there is currently keen interest in exploring this feature of the older adults' performance as a measure of interest in and of itself (e.g., Ram, Lindenberger, $\&$ Blanchard-Fields, 2009). For studies of the association between sensory and cognitive processing, age differences in within-subjects variability raise questions about the reliability of single estimates of sensory function from a small number of trials, the types of sensory acuity measures employed most frequently in prior studies. Finally, there is considerable evidence that deficits in peripheral sensory function with advancing age are more than the simple loss of acuity or sensory threshold elevation that has been measured previously. In particular, in several senses, there is evidence of decline in various aspects of sensory temporal processing with increasing age (e.g., Amberson, Atkeson, Pollack, \& Malatesta, 1979; Gordon-Salant \& Fitzgibbons, 1999; Moore, Peters, \& Glasberg, 1992; Schneider, PichoraFuller, Kowalchuk, \& Lamb, 1994; Strouse, Ashmead, Ohde, \& Granthan, 1998; Van Doren, Gescheider, \& Verrillo, 1990).

To address these shortcomings, the authors began a largescale cross-sectional laboratory study of sensory processing and cognition in adults. The following advancements were made in this project, relative to previously completed studies of this problem: (1) three senses, hearing, vision, and touch, were included; (2) rigorous, reliable, criterion-free psychophysical methods were used throughout; (3) multiple measures of acuity and temporal processing were obtained in each of the three senses; and (4) for a laboratory-based study, a relatively large sample of subjects $(N=245)$ was included. For various auditory, visual, and tactile measures, the group data and some correlational analyses for subsets of subjects have been presented elsewhere in a series of publications (Busey, Craig, Clark, \& Humes, 2010; Craig, Rhodes, Busey, Kewley-Port, \& Humes, 2010; Fogerty, Humes, \& Kewley-Port, 2010; Humes, Busey, Craig, \& Kewley-Port, 2009; Humes, Kewley-Port, Fogerty, \& Kinney, 2010). However, each of these studies presented only subsets of results focused primarily on separate modalities or tasks. A global, integrated analysis of the entire data set, with a focus on individual differences, was not possible until the completion of data collection for all subjects in all tasks. The present analysis addresses the larger issue of the relationship between the changes in sensory and cognitive functioning by examining these individual differences in a completed data set. This is the primary purpose of this article.

It should be emphasized that this project addressed possible associations among measures of sensory function and cognitive function, with the enhancements over prior studies in this area noted above. The project used measures of sensory processing that had little history in the individualdifferences literature, and thus a cross-sectional approach was deemed more efficient to address possible associations of sensory processing, cognition, and aging. It is recognized, however, that an association with aging can truly be established only with longitudinal data (e.g., Evans, 1978; Salthouse, 2010; Schaie, 1983). These cross-sectional data, however, will likely provide insights into potential associations among these phenomena to better inform and guide the development of more time-intensive longitudinal studies in the future. Of course, cross-sectional approaches not only are more efficient for exploratory studies, but also can avoid some of the potential difficulties encountered in longitudinal studies, such as practice or learning effects from repeated evaluations (e.g., Salthouse, 2010).

\section{Method}

\section{Subjects}

Three groups of adults participated in this study. The first group consisted of 50 young adults (40 females and 10 males) with a mean age of 22.6 years (range $=18-30$ years), the second group consisted of 60 middle-aged adults (39 females and 21 males) with a mean age of 48.1 years (range $=40-55$ years), and the third group consisted of 135 older adults ( 75 females and 60 males) with a mean age of 70.8 years (range $=60-87$ years). The distribution of subject ages from the youngest through the oldest is illustrated in Fig. 1. Initially, we developed the testing protocols for the older group, in order to ensure that the vast majority of older adults could complete the tasks. Recruitment of older adults preceded that of young adults, who were recruited to obtain "normative" values for each of the tasks 


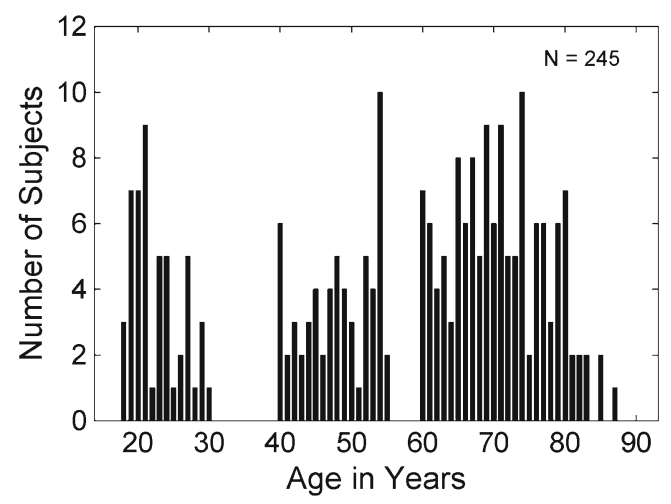

Fig. 1 The distribution of subject ages for the sample of 245 adults included in this study

in this study. Since it became apparent that there were many significant differences in performance between the young and older adults on these tasks, the middle-aged group was added to provide additional data between these two age group extremes.

Subjects were recruited for this study via advertisements in the local newspaper, bulletins or flyers for local community centers or organizations and posters at various locations on the campus of Indiana University, Bloomington. For this study, the only selection criteria were based on age (1835 years for the young adults, $40-55$ years for the middleaged adults, and 60-89 years for the older adults), a score of $\geq 25$ on the Mini Mental State Examination (MMSE; Folstein, Folstein, \& McHugh, 1975), and several measures of basic sensory acuity. Maximum acceptable hearing thresholds and allowable visual acuity were established. Specifically, subjects had to have corrected visual acuity of at least 20/40 based on an evaluation with a Snellen chart by a licensed optometrist, hearing thresholds for air-conducted pure tones that did not exceed a maximum permissible value at each of several frequencies in at least one ear, and no evidence of middle-ear pathology in the test ear (air-bone gaps less than $10 \mathrm{~dB}$ and normal tympanograms). The maximum acceptable hearing thresholds (measured clinically) were (1) $40 \mathrm{~dB}$ HL (ANSI, 2004) at 250, 500, and $1000 \mathrm{~Hz}$; (2) $50 \mathrm{~dB} \mathrm{HL}$ at $2000 \mathrm{~Hz}$; (3) $65 \mathrm{~dB} \mathrm{HL}$ at $4000 \mathrm{~Hz}$; and (4) $80 \mathrm{~dB} \mathrm{HL}$ at 6000 and $8000 \mathrm{~Hz}$. These limits were designed not to be particularly selective, but to ensure that the stimuli would most likely be visible and audible when presented on subsequent tasks. These limits were confirmed directly via identification screening. All subjects were required to pass an identification screening of the four auditory, visual, and tactile stimuli in isolation, used in subsequent temporal order and temporal-masking measures, with at least $90 \%$ accuracy on one of up to four 20 -trial blocks for the auditory and visual tasks and $80 \%$ accuracy for the tactile measures. This was to ensure that listeners would be able to complete the subsequent temporal order and temporal-masking measures, which were targeting identification performance of either $50 \%$ or $75 \%$ correct (see below). If subjects did not reach this $80 \%-90 \%$ identification accuracy criterion during screening, they were rescreened on a separate day. Subjects ultimately unable to reach this criterion were not included in this study, although many of them elected to complete measures of acuity and gap detection that did not make use of these same stimuli and their data have been included in an earlier report (e.g., Humes et al., 2009). All told, there were 81 subjects who responded to ads but, subsequently, did not meet one or more of the foregoing selection criteria: 11 young adults, 2 middle-aged adults, and 68 older adults. In 50 of the 81 cases of exclusion, the reason was pure-tone hearing loss that was too severe in the better ear, with 22 cases excluded for failing one or more of the sensory identification screenings.

Informed consent was obtained from all subjects prior to the initial screening, and subjects were paid for their participation. Young adults were paid $\$ 7$ or $\$ 8$ per hour (increased over the course of the study), whereas older adults were paid $\$ 10$ per hour. For the results reported here, the total testing time for each subject was about $60 \mathrm{~h}$, typically divided into 40 sessions, each $90 \mathrm{~min}$ in duration. With such a large amount of data collection per subject, several subjects did not complete the entire study after enrolling. To obtain complete data from the 245 subjects reported here, a total of 446 subjects were enrolled. Of the 201 who withdrew from the study at various stages, the vast majority were young adults $(N=111)$, with 30 middle-aged and 60 older adults also withdrawing from the study prior to completion. Partial data are available for subsets of test measures for the 201 subjects who withdrew from the larger study at various points in time. Some of these data have been reported previously (e.g., Busey et al., 2010; Craig et al., 2010; Humes et al., 2009). The focus here, however, is on the data from subjects who provided complete data for the entire study (with a few minor exceptions noted below). Data were available for some preliminary demographic, auditory, and cognitive measures for 163 of the 201 subjects who withdrew from the study, including 96 young adults, 22 middleaged adults, and 45 older adults. When the "withdrawals" are compared with those who completed the study within each age group, there were no significant $(p>.05)$ differences in age, hearing thresholds, or cognitive function (Verbal Comprehension, Perceptual Organization, Working Memory, and Processing Speed subscales of the WAIS-III; Wechsler, 1997). For the middle-aged and older adults, there were also no differences in gender distribution between those who withdrew and those who completed the study. However, for the young adults, the proportion of males in the withdrawal group was significantly greater $(p<.01)$ than for subjects who completed the study. From the measures 
available, the group of subjects who completed the study does not differ appreciably from the group that withdrew from the study.

\section{Procedure}

A general objective for this study was to obtain a comprehensive set of threshold sensitivity and temporal-processing measures in hearing, vision, and touch, using identical psychophysical procedures and similar stimuli for each sense. It was also important, as was noted previously, that the psychophysical measures yield criterion-free estimates of performance and do so reliably. Forced-choice adaptive (Levitt, 1971) or constant-stimuli methods were used throughout this study. Furthermore, it was also a goal to have the tasks range from simple detection tasks to closed-set identification tasks. For the closed-set identification tasks, it was decided that the complex stimuli to be identified would be brief vowels for hearing, orthographic letters in vision, and highly identifiable tactile patterns (Cholewiak \& Craig, 1984) in touch. There were four stimuli to be identified in each sense. Closed-set identification should yield reliable results but still draw on some cognitive resources in the processing of the stimuli. Thus, it was viewed as requiring higher-level processing than the other simple detection tasks employed in other measures, but not necessarily as high level as open-set recognition of stimuli.

The perceptual phenomena studied in each sense included acuity or threshold sensitivity, temporal gap detection, temporal order identification, and temporal masking. Typically, there were multiple measures of each phenomenon, but this was not always the case. For example, auditory threshold sensitivity was measured at each of three frequencies $(500,1400$, and $4000 \mathrm{~Hz})$, whereas there was only one measure of visual gap detection. The full set of 40 dependent psychophysical sensory measures in this study appears in Table 1. Importantly, for each of the measures listed in Table 1, a "threshold estimate" of performance was preceded by 20-40 familiarization trials, which included trial-to-trial feedback, and was obtained on the basis of three separate and stable blocks of trials that, when pooled, totaled 200-250 trials. The details of the stimuli and the psychophysical procedures for all of the dependent measures appearing in Table 1 have been presented in a series of prior studies, each focusing on various subsets of the entire data set (Busey et al., 2010; Craig et al., 2010; Fogerty et al., 2010; Humes et al., 2009; Humes et al., 2010).

In addition to those measures completed during the initial screening (MMSE, audiological and optometric examinations), subjects also completed the full WAIS-III. This included the 13 standard scales plus the 2 optional scales of incidental learning. Finally, all subjects completed the
Sickness Impact Profile (SIP; Bergner, Bobbitt, Carter, \& Gilson, 1981), a 136-item checklist of general health, including physical (sensory) and cognitive capabilities.

In anticipation of subject attrition, the study was divided into four phases for data collection, with psychophysical measurements completed for each of the three senses in each phase. This increased the likelihood of obtaining at least some data from all three senses in the event of a subject's withdrawal from the study. Following screening, the subjects completed the WAIS-III and the SIP. Next, for phase 1 of the study, the measures of threshold sensitivity and gap detection (Table 1) were completed, beginning with the auditory measures, followed by tactile measures, and concluding with the visual measures. Within each sense and type of measurement, the sequence of tests was also fixed. For example, for auditory threshold sensitivity measurement, measures were obtained first at $500 \mathrm{~Hz}$, then at $1400 \mathrm{~Hz}$, and finally at $4000 \mathrm{~Hz}$. Thus, the order of appearance of each task in Table 1 also represents the fixed order of testing for all subjects in this study. For studies of individual differences, as in this study, it is common to fix the order of testing to minimize the variance introduced by varied orders of presentation across subjects. Of course, when doing so, order effects, such as practice and learning, may affect the observed performance levels over the course of the study. This use of a fixed order reinforced the need for familiarization trials prior to each measure and for stable threshold estimates based on 200-250 trials.

Phase 2 consisted of the temporal order identification measures. For each sense, four temporal order identification tasks were completed. Three of the four tasks required the identification of two-item sequences (out of the four possible stimuli), and one required the identification of a fouritem sequence. The 3 two-item sequences differed with regard to how the stimuli were presented to the subject: Both stimuli in the sequence were presented either to the same receptor (ear, finger, or visual receptive field) or to different receptors. This manipulation was designed to explore lower level (peripheral) versus higher level (central) sensory temporal-processing mechanisms. For example, for the auditory two-item, different- receptor (dichotic) task, the two sensory inputs cannot interact until the first auditory center in the brainstem processes inputs from both ears (the superior olivary complex). On the other hand, the samereceptor version of this task makes it possible for interaction of the two stimuli in the sequence at a much lower level, as low as the sensory organ for hearing (the cochlea). For the two different-receptor, two-item tasks, the difference between them was in the subject's response. In one case, the subject was required to identify the stimulus sequence, just as in the same-receptor version of this task, whereas in the other case, the task was simply to identify which side (right or left) was stimulated first. The latter temporal order 
Table 1 Listing and brief description of the 40 psychophysical measures of sensory processing included in this study, organized by the phase of the study in which the measures were completed

Study Dependent measure (\# of measures)

phase

Phase 1 Auditory thresholds for pure tones: 500, 1400, and $4000 \mathrm{~Hz}$ (3)

Auditory gap detection thresholds for 1000-Hz wide bands of noise centered at either 1000 or $3500 \mathrm{~Hz}$ and presented at $91 \mathrm{~dB}$ SPL (2)

Visual flicker-fusion thresholds: flicker rates of $2,4,8$, and $32 \mathrm{~Hz}$ at mean luminance level of $127.5 \mathrm{~cd} / \mathrm{m}^{2}$ (4)

Visual gap detection threshold (1)

Tactile thresholds for sinusoidal vibration: 30 and $250 \mathrm{~Hz}(2)$

Tactile gap detection thresholds for $30-\mathrm{Hz}$ wide bands of noise centered at either 30 or $250 \mathrm{~Hz}$ and presented at $25 \mathrm{~dB}$ sensation level (2)

Phase 2 Auditory two-item same-ear vowel identification, four-item same-ear vowel identification, two-item different-ear vowel identification, two-item different-ear location identification; 70-ms vowels low-pass filtered at $1800 \mathrm{~Hz}$ and presented at $83 \mathrm{~dB}$ SPL (4)

Visual two-item same-field letter identification, four-item same-field letter identification, two-item different-field letter identification, two-item different-field location identification for 30 -ms letter stimuli presented at $\geq 1.16$ contrast ratio re: a $40 \mathrm{~cd} / \mathrm{m}^{2}$ background (4)

Tactile two-item same-finger pattern identification, four-item same-finger pattern identification, two-item different-finger (right and left index finger) pattern identification, two-item different-finger location identification; 26-ms moderate-intensity tactile patterns presented on an array of vibratory pins as stimuli (4)

Phase 3 Auditory temporal masking of vowel identification: 2 signal-to-masker amplitude ratios $\times 2$ masker types (noise, babble-like) $\times 2$ types of temporal masking (forward, backward); same target stimuli as phase 2, but vowel duration decreased to $40 \mathrm{~ms}$ (8)

Visual temporal masking of letter identification: one forward masking, one backward masking; same target stimuli as in phase 2 (2)

Tactile temporal masking of pattern identification: one forward masking, one backward masking; same target stimuli as in phase 2 (2)

Tactile spatial acuity: right index finger (2)

identification task was included because this is most often considered the temporal-order judgment in the long history of interest in this measure (e.g., Fraisse, 1984; James, 1890). Finally, the four-item sequence was included to increase the cognitive demands for this temporal order identification task, thereby increasing the likelihood for uncovering a common underlying cognitive factor. For all of the phase 2 measures, the threshold estimate obtained was the stimulus onset asynchrony (SOA) that was approximately midway between chance and $100 \%$ correct performance on the psychometric function relating performance to SOA. Further details regarding the stimuli and procedures for phase 2 measures can be found elsewhere (Busey et al., 2010; Craig et al., 2010; Fogerty et al., 2010; Humes et al., 2010).

Phase 3 consisted of all of the temporal-masking measures, plus measures of tactile spatial acuity (right index finger). The latter was a late addition to the study protocol and, although more like measures of threshold sensitivity in phase 1, was added to the end of phase 3 prior to commencing that portion of the study. Regarding the primary focus of phase 3, unlike most prior studies of temporal masking that have focused on stimulus detection preceding or following a masker, the temporal-masking task in this study was the closed-set identification of one of the four possible stimuli. SOA threshold estimates were obtained for one forwardmasking and one backward-masking condition, using a noise-like masker in vision and in touch. For the auditory measurements, two signal-to-masker amplitude ratios were employed for each of two masker types (noise and a babble-like mix of the vowels) in each of the two temporal-masking conditions (forward and backward), resulting in a total of eight dependent measures included here. Again, further details regarding the phase 3 stimuli and measures can be found elsewhere (Humes et al., 2010).

\section{Data analyses}

In phases 2 and 3, some subjects were unable to perform the tasks above chance performance levels even at the longest SOAs, despite several (four to six) immediate repetitions of the task and despite pilot testing all of the psychophysical measures prior to finalizing various paradigm parameters. When subjects were unable to perform above chance, a value corresponding to "could not do task" was entered to represent the fact that the subject could not perform the task. In addition to these data entries, if the data to be entered were actually missing at the time of data entry-typically, due to research assistant or protocol-monitoring error-a value corresponding to "missing data" was entered.

An analysis of the "could not do task" data entries revealed no such entries for any of the phase 1 threshold 
sensitivity and gap detection measures and very few for any of the phase 3 temporal-masking measures (six cases for visual forward masking, three cases for visual backward masking, and one for each of tactile forward masking, backward masking, and spatial acuity). However, several of the phase 2 temporal order-identification measures had entries of "could not do task," indicating that it was not possible to obtain a valid threshold SOA estimate despite repeated attempts to do so. Specifically, the subjects could not perform the task above chance levels of performance when using the maximum SOA available. A detailed breakdown of the number of subjects for whom this occurred for each of the phase 2 dependent measures is provided in Table 2. For all but four of these phase 2 measures, the entry of "could not do task" was relatively rare, occurring for fewer than about $7.5 \%$ of the subjects (18 or fewer occurrences). For the auditory four-item temporal order task, however, the maximum SOA was insufficient to reach threshold for 41 subjects, 20 of whom were older adults, 15 middle-aged adults, and 6 young adults. For the corresponding tactile four-item temporal order task, there were 70 subjects for whom an SOA could not be measured even for the maximum SOA value. Of these 70 subjects, 61 were older adults and 9 were middle-aged adults. For the 2 two-item tactile pattern identification tasks, 46 (44 older adults) reached the maximum SOA limit for the samereceptor version of the task, and 64 (60 older adults) reached

Table 2 Number of occurrences of "could not do task" data entry values for each of the 12 psychophysical temporal order identification measures from phase 2 of the study

Dependent measure

Number of subjects with "could not do task" values

\begin{tabular}{ll}
\hline Auditory & \\
Two-item same-ear vowel identification & 2 \\
Four-item same-ear vowel identification & 41 \\
Two-item different-ear vowel identification & 8 \\
Two-item different-ear location identification & 14 \\
Visual & \\
Two-item same-field letter identification & 3 \\
Four-item same-field letter identification & 5 \\
Two-item different-field letter identification & 18 \\
Two-item different-field location identification & 1 \\
Tactile & \\
Two-item same-finger pattern identification & 46 \\
Four-item same-finger pattern identification & 70 \\
Two-item different-finger pattern identification & 64 \\
Two-item different-finger location identification & 6 \\
\hline
\end{tabular}

Note. The few remaining occurrences of such data entry values are described in the text. the maximum SOA limit for the different-receptor version of the task. Interestingly, note that when the same two-item different-receptor stimuli are presented but the response is changed to identifying the location (right or left index finger), rather than the stimulus pattern, only 6 of the 245 subjects had an SOA that exceeded the limits in the software.

For those subjects with "could not do task" values, all that is known is that their SOA value exceeded the maximum value included in the protocol software. This maximum was established on the basis of preliminary pilot data, as has been noted, but also some consideration of memory constraints for the temporal order identification tasks. Clearly, the longer the SOA, the more difficult it would be to remember and subsequently identify the stimulus sequence. For the tactile temporal order tasks, the maximum SOA was $3,000 \mathrm{~ms}$, whereas the maximum for the auditory four-item temporal order task was $1,500 \mathrm{~ms}$. For subsequent data analysis, all of the "could not do task" data entries for these 40 dependent measures were replaced with numerical values of 10,000 . As a result, subsequent data analyses for the entire data set were based on assigned ranks for each of the 40 dependent variables and 245 subjects. The validity of this approach for subsequent correlation-based PC factor analyses of the data set was evaluated and is described in detail below.

With regard to missing data (data entry values of "missing data"), 36 of the 40 dependent measures had data missing from 4 or fewer of the 245 subjects. The four dependent measures for which more data were missing were the two visual temporal-masking measures (20 missing data values for each) and the two tactile temporal-masking measures (18 and 23 missing data values for forward and backward masking, respectively). These dependent measures were the last measures from phase 3 to be obtained and represent subject attrition in the final stages of the protocol. For each of these four dependent measures, there was a roughly equal distribution of missing values across the young, middle-aged, and older adults.

After substituting the constant " 10000 " for all "could not do task" entries in the data matrix, each variable was separately rank-transformed, with high ranks reflecting poor psychophysical performance (high thresholds, large SOAs, etc.). Ties were resolved by assigning the average rank to a group of tied values. Because observations were not partitioned by age group before transformation, the rank-ordered data preserve information about relative performance on each measure across age groups, as well as correlation structure across variables within each age group. Missing ranks were then replaced using multivariate imputation by chained equations (MICE; van Buuren \& GroothuisOudshoorn, 2011; van Buuren \& Oudshoorn, 2000). Specifically, predictive mean matching (Little, 1988) was used 
to impute the missing rank data on each incomplete variable from all available predictors. Each missing rank was then replaced with the arithmetic mean of five synthetically generated ranks.

Finally, the imputed rank order matrix served as data input to a correlation-based PC analysis. Applying PC extraction to ranks yields a nonmetric factor structure that is invariant under monotone transformations of the data within each column and closely approximates the structure recovered using standard (metric) factor analysis when the monotone relationships are strictly linear (Kruskal \& Shepard, 1974; Woodward \& Overall, 1976). The validity of the conversion to ranks was evaluated using the subset of data for which there were no missing observations $(n=186)$ and applying PC extraction to both the (1) rank-transformed data and (2) Spearman's rank correlation matrix. The solutions recovered separately for three to eight dimensions showed no appreciable differences in the number of significant eigenvalues, in the magnitude or pattern of factor loadings, or in the fit statistics.

\section{Results and discussion}

As has been noted, the group results, as well as some correlational analyses, for various subsets of the data set have been presented in a series of prior publications (Busey et al., 2010; Craig et al., 2010; Fogerty et al., 2010; Humes et al., 2009; Humes et al., 2010). The focus here is on the individual differences, using factor analysis of the entire data set. A thorough analysis of the individual differences in sensory processing, across all sensory tasks and all three senses, and cognitive processing, as well as the association of each type of processing with age, has not been presented previously for the data gathered in this large project. Two primary sets of dependent measures were obtained from all 245 subjects in this study: (1) 40 psychophysical measures of threshold sensitivity and temporal processing in hearing, vision, and touch and (2) 15 measures of cognitive function from the WAIS-III. PC factor analyses were conducted separately for each of these two sets of dependent measures. After reducing the redundancy in each of these sets of dependent measures via PC analysis, the association of the factors that emerged from these analyses with age was then examined.

Factor analysis of the psychophysical measures

For the 40 dependent measures of threshold sensitivity and temporal processing, $\mathrm{PC}$ analysis of the correlation matrix using an eigenvalue $>1$ criterion resulted in the identification of eight underlying PCs. The Kaiser-Meyer-Olkin (KMO) measure of sampling adequacy was high (.92), and
Bartlett's test of sphericity was significant $(p<.001)$. An eight-factor solution accounting for $69.7 \%$ of the total variance was obtained. All 40 of the communality values were greater than .5 , with $20 \%$ between .50 and $.59,25 \%$ between .60 and $.69,42.5 \%$ between .70 and .79, and $12.5 \%$ between .80 and .89 . Thus, all 40 dependent measures were reasonably represented in the factor solution. The resulting pattern matrix from the oblique-rotated solution is shown in Table 3. On the basis of the component weights in this matrix, each of the eight principal components was interpreted and labeled as follows: (1) auditory temporal order and temporal masking (audTOTM); (2) tactile temporal order and temporal masking (tacTOTM); (3) auditory and tactile threshold sensitivity (audtacTHR); (4) visual flicker-fusion threshold sensitivity (visFF); (5) auditory, visual, and tactile temporal order location (audtacvisTOloc); (6) visual temporal order and temporal masking (visTOTM); (7) auditory gap detection (audGD); and (8) tactile gap detection (tacGD). Note that although the second component is interpreted and labeled as tactile temporal order and temporal masking for simplicity, the tactile spatial acuity measure was also highly weighted on this factor, but tactile threshold sensitivity for sinusoidal vibration was not. Rather, the latter measure was associated with the audtacTHR factor. Furthermore, note that visual gap detection is the only dependent measure that was not weighted at least moderately on any of the eight factors identified. Although the communality value for this variable was reasonable (.52), it was also the lowest value among the 40 dependent measures.

Several observations can be made regarding the factors that emerged from this analysis. First, typically, there is a separation between types of tasks, with phase 1 signal detection or gap detection tasks associated with one set of factors (audtacTHR, visFF, audGD, tacGD) and phase 2 and phase 3 temporal order and temporal-masking closed-set identification tasks associated with another set of factors (audTOTM, tacTOTM, visTOTM, audtacvisTOloc). Second, within a sensory system, there were strong associations between the various temporal order and temporal-masking measures from phases 2 and 3 (audTOTM, tacTOTM, visTOTM). Third, only two of the eight factors are crosssensory factors (audtacTHR and audtacvisTOloc). For the five dependent measures entering into the audtacTHR factor, the correlations (Spearman rho) among the three auditory dependent measures (hearing thresholds at 500, 1400, and $4000 \mathrm{~Hz}$ ) ranged from .55 to .70 ; between the two tactile vibratory thresholds ( 30 and $250 \mathrm{~Hz}$ ), the correlation was .62; and for the six cross-sensory correlations, the values ranged from .37 to .55 , with the highest crosssensory correlation (.55) being between the hearing threshold at $4000 \mathrm{~Hz}$ and the tactile threshold at $250 \mathrm{~Hz}$. For the three temporal order location measures, the correlations 
Table 3 Pattern matrix showing component weights from eight-factor principal-component analysis of the 40 psychophysical measures of threshold sensitivity and temporal processing in hearing, touch, and vision (Table 1)

\begin{tabular}{|c|c|c|c|c|c|c|c|c|}
\hline \multirow[t]{2}{*}{ Dependent measure } & \multicolumn{8}{|c|}{ Principal component } \\
\hline & audTOTM & tacTOTM & audtacTHR & visFF & audtacvis TOloc & visTOTM & audGD & tacGD \\
\hline audTHR500 & .018 & .107 & .755 & .084 & -.111 & -.147 & .063 & .045 \\
\hline audTHR1400 & .130 & -.047 & .900 & -.058 & -.135 & -.081 & -.005 & .102 \\
\hline audTHR4000 & .105 & .134 & .791 & -.069 & -.039 & -.131 & .077 & -.068 \\
\hline audGD1000 & .026 & -.222 & .098 & .216 & .147 & -.070 & .761 & .123 \\
\hline audGD3500 & -.010 & -.187 & .187 & -.003 & .177 & -.075 & .789 & .145 \\
\hline visFF2 & -.004 & .017 & -.129 & .878 & -.005 & .009 & .166 & .043 \\
\hline visFF4 & -.097 & .106 & -.083 & .963 & -.095 & -.041 & .101 & .055 \\
\hline visFF8 & -.023 & -.002 & .086 & .942 & -.165 & -.005 & -.015 & -.034 \\
\hline visFF32 & .209 & -.225 & .253 &.$\underline{414}$ & .246 &.$\underline{353}$ & -.212 & -.208 \\
\hline visGD & .005 & .175 & .092 & .196 & .141 & .060 & .157 & .280 \\
\hline tacTHR30 & -.107 & .120 & .669 & .006 & .099 & -.170 & .200 & -.249 \\
\hline tacTHR250 & -.004 & .131 & .685 & -.051 & .165 & -.085 & .022 & -.277 \\
\hline tacGD30 & .066 & .253 & -.280 & -.003 & -.084 & -.105 & .178 & .724 \\
\hline tacGD250 & .088 & -.130 & -.005 & .033 & .243 & -.029 & .084 & .719 \\
\hline audTO_2same & .538 & .189 & .035 & .086 & .133 & -.098 & .210 & -.111 \\
\hline audTO_4same & .340 & .130 & -.325 & -.022 & .361 & -.078 & .319 & -.239 \\
\hline audTO_2diff & .475 & .258 & -.243 & .001 & .320 & -.002 & .103 & -.159 \\
\hline audTO_2diffloc & .257 & -.090 & -.234 & -.042 & .683 & -.005 & .098 & .070 \\
\hline visTO_2same & .057 & .252 & .168 & .018 & .087 & .413 & .126 & .005 \\
\hline visTO_4same & .026 & .266 & .149 & .045 & .107 & .446 & .056 & .057 \\
\hline visTO_2diffloc & -.134 & .047 & .326 & -.106 & .659 & .031 & .092 & .100 \\
\hline visTO_2diff & -.099 & .188 & .194 & .015 & .387 & .223 & .004 & .150 \\
\hline tacTO_2same & -.002 & .933 & -.008 & .035 & .000 & .047 & -.106 & .023 \\
\hline tacTO_4same & .082 & .867 & .064 & -.035 & .026 & .032 & -.114 & .006 \\
\hline tacTO_2diff & -.035 & .871 & .062 & -.042 & .046 & .063 & -.056 & -.004 \\
\hline tacTO_2diffloc & -.102 & .166 & .045 & -.125 &.$\underline{778}$ & -.137 & .163 & .037 \\
\hline $\operatorname{tacSp} \_1$ & .035 & -.731 & .038 & .000 & -.090 & -.071 & .108 & -.105 \\
\hline tacSp_2 & .081 & -.749 & -.093 & -.121 & .036 & .007 & .067 & .038 \\
\hline visFM & .062 & .095 & -.324 & .049 & -.095 & $\underline{.939}$ & -.199 & -.146 \\
\hline visBM & .004 & -.157 & .072 & .164 & .039 & -.613 & -.163 & -.139 \\
\hline tacFM & .057 & .849 & .125 & .042 & -.079 & -.004 & -.119 & .044 \\
\hline tacBM & .047 & .758 & .063 & -.032 & .012 & .062 & -.022 & -.007 \\
\hline audFMbab1 & .713 & .108 & .103 & .024 & -.316 & .017 & .251 & .026 \\
\hline audFMbab2 & .696 & .143 & .065 & .032 & -.213 & .017 & .249 & -.024 \\
\hline audBMbab1 & -.803 & -.102 & -.070 & -.068 & -.165 & .272 & .275 & -.120 \\
\hline audBMbab2 & $\underline{-.798}$ & -.091 & -.101 & -.044 & -.158 & .283 & .250 & -.138 \\
\hline audFMns1 & .787 & -.130 & .169 & -.045 & -.173 & .178 & .059 & -.048 \\
\hline audFMns2 & .728 & -.074 & .233 & -.188 & -.131 & .193 & .055 & -.020 \\
\hline audBMns1 & -.894 & .163 & .077 & .030 & -.133 & -.116 & .075 & -.095 \\
\hline audBMns2 & -.833 & .089 & .108 & .057 & -.125 & -.161 & .007 & -.083 \\
\hline
\end{tabular}

Note. Component weights $\geq 0.3$ are highlighted via bold font and underscore. Labels, explained more fully in the text, are: $\mathrm{A}=$ auditory, $\mathrm{T}=$ tactile, $\mathrm{V}=$ visual, $\mathrm{THR}=$ threshold, $\mathrm{FF}=$ flicker fusion, $\mathrm{GD}=$ gap detection, $\mathrm{TO}=$ temporal order, $\mathrm{TM}=$ temporal masking, $\mathrm{BM}=$ backward masking, $\mathrm{FM}=$ forward masking, $\mathrm{Sp}=$ spatial acuity, $\mathrm{bab}=$ babble-like masker, $\mathrm{ns}=$ noise masker.

(Spearman rho) among these measures ranged from .41 to .59. It is noteworthy that when the auditory, visual, and tactile two-item sequences remained the same but the task was changed from identification of the sequence of stimuli 
(vowels, patterns, letters) to the sequence of locations (right, left), the cross-sensory correlations increased to the extent that a separate audtacvisTOloc factor emerged for the location task. As was noted previously, the location order task is the classic implementation of temporal order judgments (e.g., Fraisse, 1984; James, 1890), and the identification of "where" appears to be a task that is not sensory system specific.

An oblique Promax rotation (kappa $=4$ ) was employed to facilitate the foregoing interpretation of the PC factor analysis, and moderate correlations among some of the eight factors were observed. Table 4 presents the matrix of Pearson $r$ correlations among the eight components. Several factors are moderately correlated, with 12 of the 28 component correlations exceeding a value of .40 . In general, the two factors with the weakest cross-component correlations are visTOTM and tacGD.

Given the foregoing matrix of component correlations, a second-order PC factor analysis was performed on the eight factor scores (Gorsuch, 1983). A stopping criterion of eigenvalue $<1.0$ was again employed, and one factor emerged, accounting for $44.6 \%$ of the variance. The KMO sampling adequacy measure was again good (.84) and Bartlett's test of sphericity was significant $(p<.001)$. The communality values for this onefactor solution were moderate (.29-.67) for seven of the eight factors but was weak (.18) for the tacGD factor. This single common factor is interpreted here as a global sensoryprocessing (SensProc_Global) factor. The component weights for each of the eight first-order factors on this second-order SensProc_Global factor were as follows: (1) audTOTM $=.76$, (2) tacTOTM $=.82$, (3) audtacTHR $=.72$, (4) visFF $=.54$, (5) audtacvisTOloc $=.75,(6)$ visTOTM $=.66,(7)$ audGD $=.57$, and $(8)$ tacGD $=.43$.

Factor analysis of the cognitive measures

The 13 standard scales of the WAIS-III, plus the two optional incidental-learning scales, were obtained from all 245 subjects in this study. Five subjects were missing scores for the two incidental-learning scales, and 1 subject was missing a score for the symbol search scale. Given the sparse missing values, rather than employing a complex imputation procedure, the few missing values were simply replaced with the mean value prior to analysis. Raw scores from the WAIS-III scales were analyzed, rather than using standardized or age-normed scores, since this would preserve the anticipated age-related changes in cognition.

The 15 WAIS-III scale scores were subjected to a PC factor analysis using an eigenvalue $<1$ stopping rule. Three factors emerged, accounting for $64.3 \%$ of the total variance. The KMO sampling adequacy measure was very good (.87), and Bartlett's test of sphericity was significant $(p<.001)$. Communalities ranged from .43 to .80 , with $20 \%$ falling between .40 and $.49,7 \%$ from .50 to $.59,40 \%$ between .60 and .69 , and $33 \%$ above .70 . Anticipating correlations among components, oblique rotation was employed to facilitate the interpretation of the factor structure underlying the cognitive measures.

Table 5 displays the component weights from the pattern matrix for the three-factor solution. On the basis of the observed pattern, the first factor was interpreted and labeled as a process factor (process; Salthouse, 2010) and the second as a product factor (product; Salthouse, 2010). In other cognitive ability taxonomies, these might also be labeled as fluid or nonverbal and crystallized or verbal, respectively. The third factor is interpreted as an incidental-learning factor (IncLrn). Component correlational analysis revealed that the process factor was moderately correlated with the product factor $(r=.45)$ and the IncLrn factor $(r=.43)$, but there was only a weak correlation between the product and IncLrn factors $(r=.15)$.

In view of the moderate between-component correlations, a second-order $\mathrm{PC}$ analysis with eigenvalue $<1$ stopping criterion was performed on the three cognitive factor scores. A reasonable single-factor solution emerged with the KMO sampling-adequacy statistic $=.54$, a significant Bartlett's test of sphericity $(p<.001)$, communalities of .46 (IncLrn), .49 (product), and .75 (process), and $56.7 \%$ of the total variance

Table 4 Component correlation matrix for the eight principal components resulting from the analysis of the 40 psychophysical measures of sensory processing from the 245 subjects in this study

\begin{tabular}{|c|c|c|c|c|c|c|c|}
\hline & tacTOTM & audtacTHR & visFF & audtacvis TOloc & visTOTM & audGD & tacGD \\
\hline audTOTM & .62 & .42 & .31 & .51 & .35 & .46 & .22 \\
\hline tacTOTM & & .55 & .27 & .55 & .44 & .51 & .25 \\
\hline audtacTHR & & & .31 & .46 & .52 & .25 & .22 \\
\hline visFF & & & & .44 & .23 & .20 & .27 \\
\hline audtacvis TOloc & & & & & .43 & .26 & .22 \\
\hline visTOTM & & & & & & .23 & .25 \\
\hline audGD & & & & & & & .18 \\
\hline
\end{tabular}

Note. Pearson $r$ values $\geq .4$ have been highlighted using bold font. 
Table 5 Pattern matrix showing component weights from three-factor principal-component analysis of the 15 scale scores from the WAIS-III for the 245 adults in this study

\begin{tabular}{|c|c|c|c|}
\hline & \multicolumn{3}{|c|}{ Component } \\
\hline & Process & Product & IncLrn \\
\hline Pict completion & .472 & .235 & .085 \\
\hline Vocabulary & -.015 & .890 & -.004 \\
\hline Dig symbol coding & .859 & -.233 & .087 \\
\hline Similarities & .136 & .722 & .112 \\
\hline Block design & .696 & .050 & .113 \\
\hline Arithmetic & .260 & .556 & -.126 \\
\hline Matrix reasoning & .734 & .097 & .063 \\
\hline Digit span & .787 & .107 & $\underline{-.405}$ \\
\hline Information & -.097 & .931 & -.005 \\
\hline Pict arrangement & .483 & .283 & .076 \\
\hline Comprehension & -.154 & .897 & .055 \\
\hline Symbol search & .798 & -.173 & .228 \\
\hline Letter-number seq & .819 & -.006 & -.089 \\
\hline Pairing & .197 & .057 & .715 \\
\hline Free recall & -.074 & .017 & .883 \\
\hline
\end{tabular}

Note. Component weights $\geq 0.3$ are highlighted via bold font and underscore. IncLrn $=$ incidental learning, Pict $=$ picture, Dig $=$ digit, Seq $=$ sequence

explained. This factor is interpreted as general cognitive processing and is labeled CogProc_Global. The component weights for each of the three cognitive factors on CogProc Global were .87 (process), .70 (product), and .68 (IncLrn).

\section{Associations of factors scores with age}

Figure 2 provides scatterplots of each of the eight factor scores derived from the psychophysical measures of threshold sensitivity and temporal processing, together with the second-order global sensory processing factor (SensProc_Global), as a function of age. About one third of the factor scores show a strong association with age (Pearson $r>.70$; tacTOTM, audtacTHR, and SensProc_Global), another one third show little or no association with age $(r<.35$; visFF, audGD, and tacGD), and the remaining one third fall somewhere in between $(.55<r<.60$; audTOTM, audtacvisTOloc, and visTOTM). All of the correlations with age are statistically significant $(p<.001)$, except for tacGD $(r=$ $.06, p>.05)$.

Figure 3 provides a similar set of scatterplots for the three cognitive factors that emerged from the analysis of the WAIS-III and the second-order global cognitive function factor score (CogProc Global). As was expected (e.g., Salthouse, 2010), the process factor score declines with age ( $r=$ $-.71)$, whereas the product factor score does not $(r=-.04)$. The factor score for incidental learning (IncLrn) declines with age $(r=-.45)$, as does the second-order global cognitive function factor score $(r=-.55)$. All correlations with age, except that for product, are significant $(p<.001)$.

Given that age is moderately to strongly correlated with SensProc_Global (Fig. 2) and CogProc_Global (Fig. 3), it is expected that these two factors would also be correlated. This is confirmed in Fig. 4, which provides a scatterplot of these two sets of second-order factor scores. High factor scores on SensProc_Global reflect poor sensory function (elevated thresholds, large gap detection thresholds, long SOAs), and those with poor sensory function tend to have poor cognitive function (low CogProc_Global scores reflect low scores on the WAIS-III scales). The correlation between these two global second-order factor scores is strong $(r=-.71, p<.001)$.

The foregoing correlational analyses revealed that age is moderately and significantly correlated with most of the lower order sensory and cognitive factors, as well as the higher order factors in each domain. The lower order factors appear to capture the variance that is mostly unique to each of eight sensory-processing dimensions identified or to each of the three cognitive-processing dimensions identified. The higher order factors, on the other hand, appear to capture the variance that is common across dimensions and shared within each of the respective domains. Each of the global higher order factors, SensProc_Global and CogProc_Global, is correlated with age $(r=.75$ and -.55 , respectively) and with one another $(r=-.71)$. To gain further insight into the underlying nature of these associations among the higher order global factors and age, partial correlations were also computed. Three partial correlations were computed, each examining the association between two of the three variables (SensProc_Global, CogProc_Global, Age) while controlling for the third variable. The correlation between SensProc_Global and CogProc_Global decreased slightly from $r=-.71$ to $r_{\mathrm{p}}=-.53$ when age was controlled for. Thus, there appears to be an association between global sensory processing (including measures of threshold sensitivity, temporal order identification, and temporal masking) and global cognitive processing (including measures of process, product, and incidental learning) that is independent of age. To further confirm the age independence of the association between global sensory processing and global cognitive processing, correlations and partial correlations, controlling for age, were calculated separately for each of the three age groups. Correlations of $-.62,-.51$, and -.58 and partial correlations of $-.61,-.50$, and -.46 were observed for the young, middle-aged, and elderly groups, respectively. All of these correlations were significant $(p<$ .001). Age differences across subjects, however, do tend to accentuate the association between global sensory and global cognitive processing to some degree, especially among the older adults (who also had the widest range of ages). 
Fig. 2 Scatterplots of nine factor scores, eight from the first-order analysis of the psychophysical measures of threshold sensitivity and temporal processing in hearing, touch, and vision and one second-order general sensoryprocessing factor (SensProc Global, lower right) versus subject age for the 245 adults in this study
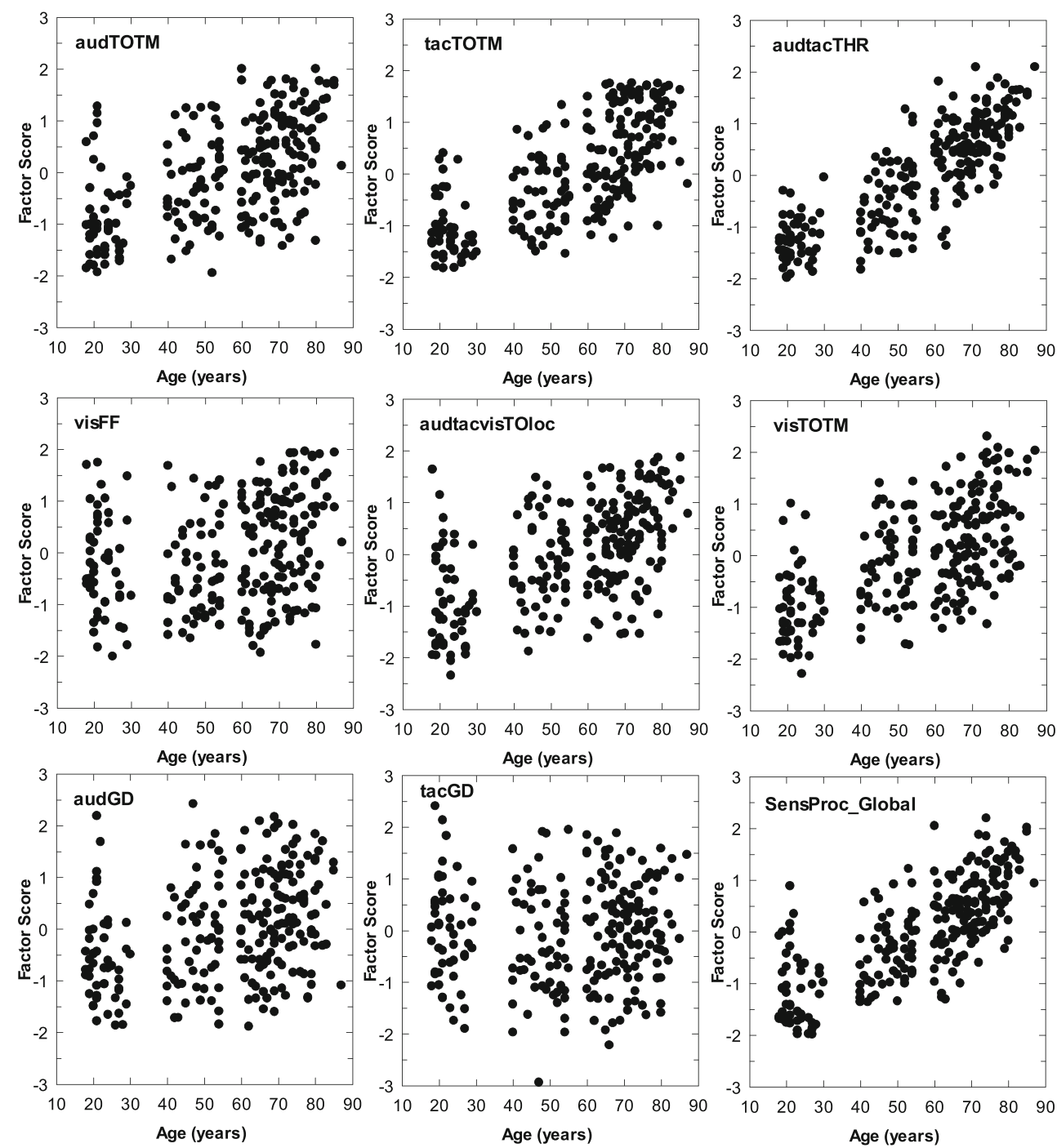

The correlation between SensProc_Global and age decreased slightly from $r=.75$ to $r_{\mathrm{p}}=.61$ when general cognitive function (CogProc_Global) was controlled for. This pattern of results suggests that sensory processing diminishes with age and this decline is largely independent of general cognitive function or the decline in general cognitive function with age.

Finally, and perhaps most interesting, when global sensory processing (SensProc_Global) is controlled for, the correlation between age and general cognitive function disappears. That is, the initial uncontrolled correlation between age and cognition was moderate, negative, and significant $(r=-.55, p<.001)$, but controlling for SensProc_Global reduces the correlation to $r_{\mathrm{p}}=-.05(p>.05)$.

Taken together, the foregoing PC analyses and subsequent correlation analyses suggest that there is an association between age and general sensory processing, when measured in multiple senses and using a range of measures from simple measures of acuity or threshold sensitivity to more complex temporal order and temporal-masking tasks.
The trends in these data indicate that the older the adult, the greater the sensory decline. That is, aging produces threshold elevations and prolonged SOAs in temporal-order identification and temporal-masking tasks. Elevated sensory thresholds, in more than one sense (primarily in hearing and touch in this study), and difficulties processing fast sequences of stimuli in more than one sense (tactile, auditory and visual, in this study), in turn, are associated with poorer cognitive function, regardless of age. This, in turn, implies that the frequently observed association between chronological age and cognition (e.g., Salthouse, 2010) may be mediated by global sensory declines of the type measured here. Of course, correlations cannot establish cause.

Associations among the various measures obtained in this study were also examined using structural equation modeling (SEM). A series of models were evaluated, and, because the results were entirely consistent with the preceding PC and correlational analyses, the SEM analyses are only summarized briefly here. 

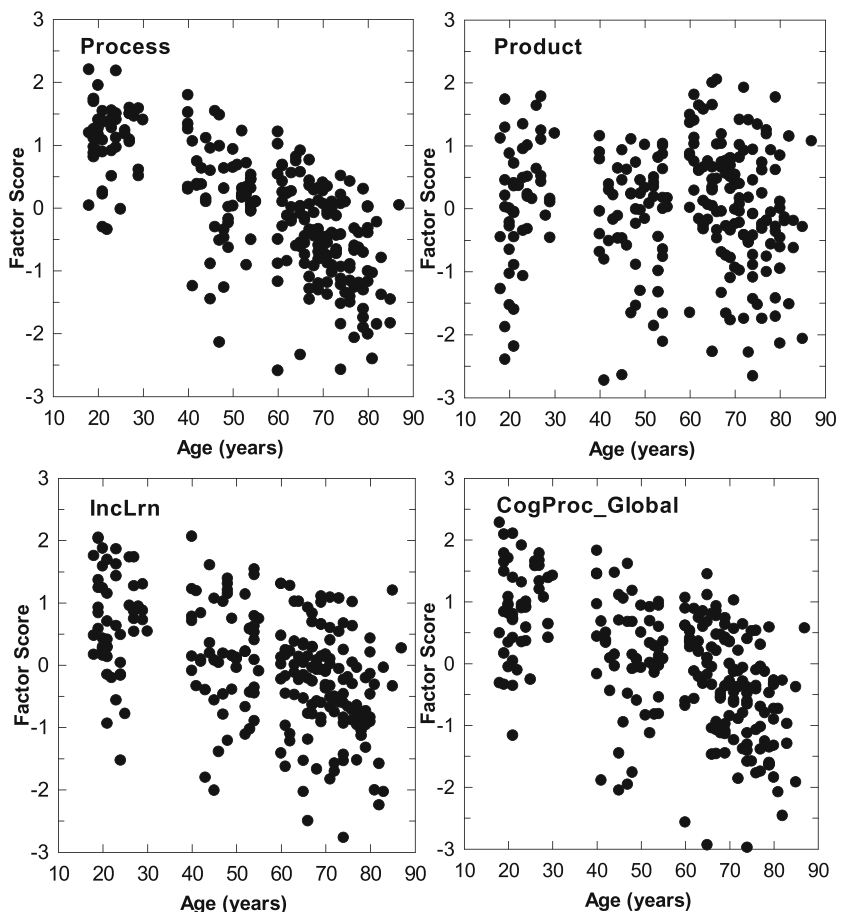

Fig. 3 Scatterplots of four factor scores, three from the first-order analysis of the WAIS-III raw scale scores and one second-order general cognitive-processing factor (CogProc_Global, lower right) versus subject age for the 245 adults in this study

Structural equation modeling

The results from the prior partial correlations suggest that when the contributions of the sensory measures are

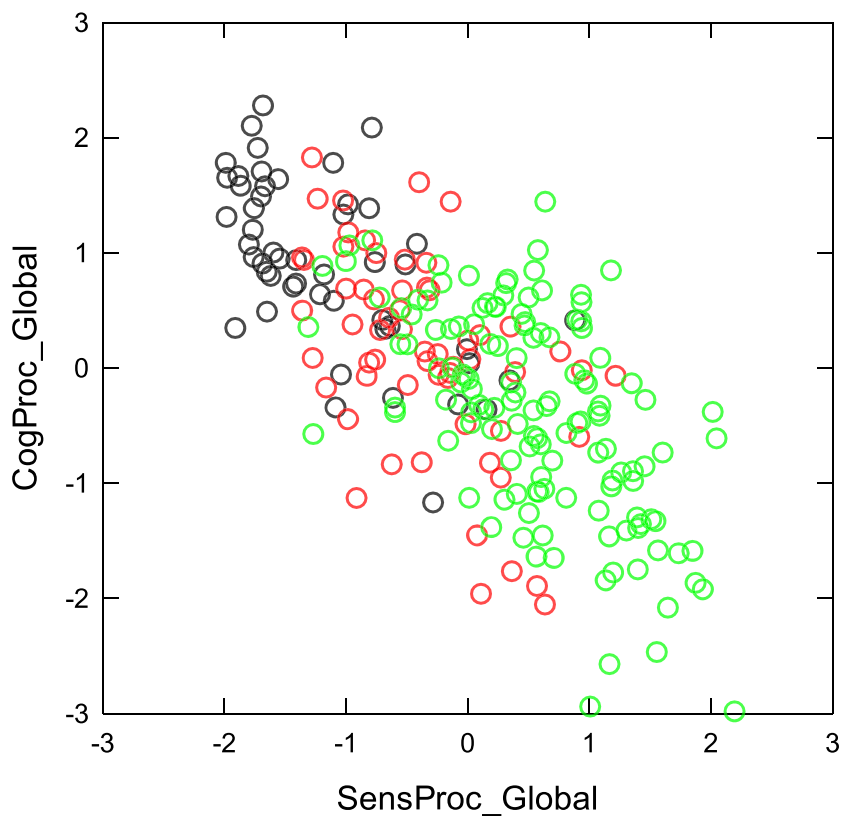

Fig. 4 Scatterplot of the higher order cognitive-processing factor score (CogProc_Global) versus the higher order sensory-processing factor score (SensProc Global) for the 245 subjects in this study. Symbols are color-coded by age group (young, black; middle-aged, red; older, green) statistically controlled for, the relation between cognition and age is quite small. This is a surprising finding, because it suggests that prior associations between cognition and aging may be mediated in part by sensory processes. To simultaneously address the relations among sensation, cognition, and aging in an alternative analysis, several structural equation models were constructed and evaluated.

As input to the structural model, the data from all 245 subjects were used, including all three age groups. As a means to stabilize the variance and equate the scales of the different measures, a Blom transform was applied to the raw measures. This essentially ranks the scores (with missing/ could-not-perform set as the highest rank and missing-atrandom left blank) and then inverse-normal transforming the ranks. The full-information maximum-likelihood analysis in AMOS (Version 21.0, SPSS Inc.) was used, which handles missing-at-random values and estimates intercepts.

The first step in the SEM analysis was to construct the measurement model for the sensory measures. After that, the cognitive measures, as estimated from the WAIS, were added to the model, and then the relation of each underlying factor with age was examined. The measurement model for the sensory measures required grouping the 31 sensory measures into subgroups on the basis of prior results with the factor analysis, as well as consideration of the tasks and modalities. This yielded 8 different latent measures, as listed in Table 6.

The measurement model fit reasonably well. The RMSEA was 0.064 , with a $90 \%$ confidence interval of $0.058-0.071$. The model has 426 degrees of freedom, and although the RMSEA is within a reasonable range, there was sufficient power in these data to reject the model $(p<.001)$. The CMIN is 856.1 , the $\mathrm{BCC}$ is $1,088.6$, and the model has an NFI value of .830 . Table 6 also shows the regression parameter estimates of the different factors with the latent global sensory factor. All eight measures produced statistically significant contributions to the latent factor, although as illustrated by the squared multiple correlations (percent variance accounted for), some measures were more strongly associated with the global sensory latent factor than were others. Measurement models without these low-weighted components were not demonstrably better when used in the models reported below.

Having estimated the global sensory-processing factor with the preceding measurement model, the WAIS measures were introduced into the model using a hierarchical model in which three latent factors were estimated using the Blom transformed, non-age-corrected raw WAIS scores. The 14 WAIS measures were grouped into product, process, and incidental-learning factors, similar to how this was done for the PC analysis reported earlier. Each of these latent cognitive factors was then used to estimate an underlying global cognitive measure. This allows us to directly estimate the 
Table 6 Regression weights for the measurement model for the global sensory factor

\begin{tabular}{|c|c|c|c|c|c|c|}
\hline Latent factor & $\begin{array}{l}\text { Standardized } \\
\text { regression weight }\end{array}$ & Estimate & S.E. & C.R. & $p$ & $\begin{array}{l}\text { Squared multiple } \\
\text { correlations }\end{array}$ \\
\hline Visual flicker fusion & .405 & 0.439 & .081 & 5.455 & $* * *$ & .164 \\
\hline Auditory temporal order/temporal masking & .803 & 1.041 & .102 & 10.185 & $* * *$ & .645 \\
\hline Tactile temporal Order/temporal masking & .866 & 1.093 & .096 & 11.412 & $* * *$ & .750 \\
\hline Visual temporal order/temporal masking & .881 & 1.132 & .103 & 11.003 & $* * *$ & .776 \\
\hline Auditory/visual/tactile Location judgment & .870 & 1.000 & (fixed) & & & .757 \\
\hline Auditory/tactile threshold & .759 & 0.873 & .097 & 8.961 & $* * *$ & .577 \\
\hline Tactile gap & .510 & 0.409 & .100 & 4.112 & $* * *$ & .260 \\
\hline Auditory gap & .656 & 0.741 & .101 & 7.322 & $* * *$ & .430 \\
\hline
\end{tabular}

Note. Each of eight latent factors was estimated by 2-6 raw scores and then regressed against the global sensory factor to produce a single estimate of global sensory performance for each subject, $* * * p<.001$.

relation between the global sensory and global cognitive latent factors, as well as the relation of each with age.

Figure 5 summarizes the results of the modeling, including the relevant regression weights/correlations and the fit statistics of four different models. In Fig. 5a, the relation between the global sensory factor and age was estimated directly, without relations between global cognition and the other two measures. The standardized regression weight between the global sensory factor and age was quite high $(.85 ; p<.001)$ and accounts for $72.4 \%$ of the variance in age. In Fig. 5b, with only the global cognitive factor predicting age, this measure also has a high regression weight $(-.756, p<.001)$ and accounts for $57.1 \%$ of the variance in age. Thus, while both latent factors are related to age, the relation seems stronger for the global sensory factor, and this factor accounts for more variability in the age measure.

Fig. $5 \mathrm{c}$ introduces both regression terms to the model and illustrates that when both global sensory and global cognitive factors were allowed to predict age, the regression weight for the global sensory factor was larger (.78 vs.
Fig. 5 Four structural models exploring the relation between the global sensory, global cognitive, and age measures. a Only the global sensory factor is regressed against age. b Only the global cognitive factor is regressed against age. $\mathbf{c}$ Both global sensory and global cognitive factors are regressed against age. $\mathbf{d}$ A correlation is introduced between the two global factors. See the text for details of fits and parameter estimates
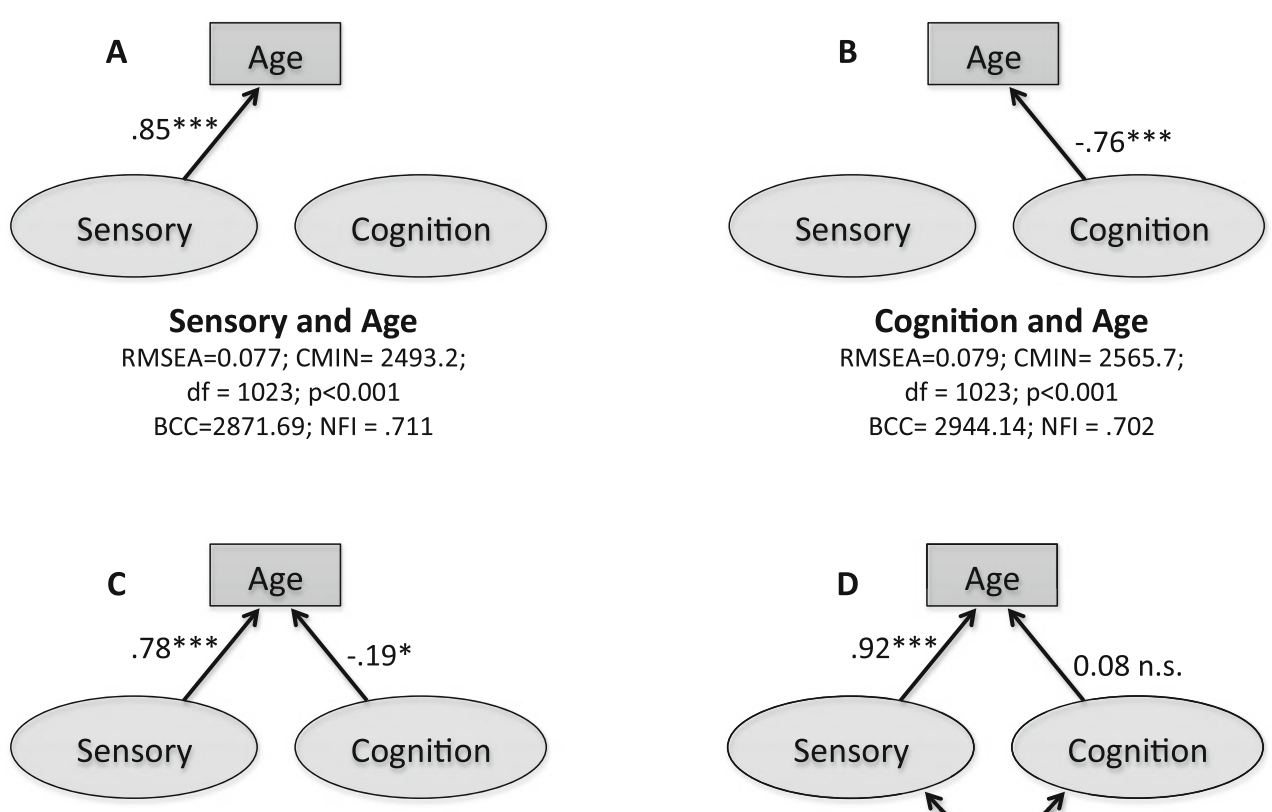

Both Sensory and Cognition
With Age

RMSEA $=0.077 ; C M I N=2487.7$;

$\mathrm{df}=1022 ; p<0.001$

$B C C=2868.6 ; \mathrm{NFI}=.711$

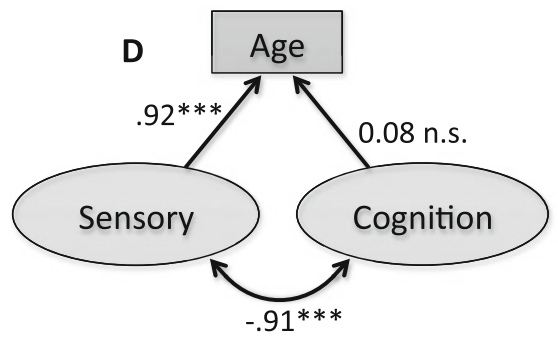

Correlation Between Sensory and Cognition

RMSEA $=0.069 ; \mathrm{CMIN}=2218.3$; $\mathrm{df}=1021 ; p<0.001$

$\mathrm{BCC}=2601.7 ; \mathrm{NFI}=.743$ 
-.19), and the model accounts for $64.5 \%$ of the variance in age. Finally, in Fig. 5d, a correlation was introduced between the global sensory and global cognitive factors. In this model, only the relation between the global sensory factor and age had a regression weight that was statistically significantly different from zero $(.92, p<.001)$, whereas the cognitive/age relation was not $(-.08 ; p>.05)$. With this last model, $72.2 \%$ of the variance in age has been accounted for, very similar to what was predicted on the basis of the global sensory factor alone, which is not surprising given the large $(-.91)$ correlation between the global sensory and global cognitive factors. Note that this last model also has the lowest $\mathrm{BCC}$ value, a value lower than the version of the model with only the global sensory factor regressed against age (Fig. 5a).

The models illustrated in Fig. 5 confirm the general findings from the factor analysis: The relation between global sensory processing and age is stronger than the relation between global cognitive processing and age. In fact, when both global factors are included in the model, the contribution from the cognitive factor is no longer statistically significantly different from zero. This is similar to the conclusion drawn from the partial-correlation analyses presented previously. These conclusions are similar despite the fact that we relied on Blom-transformed data and a fullinformation maximum-likelihood approach to the replacement of missing data for the structural model, rather than simple ranked raw data and MICE-imputed raw scores for the factor analysis.

\section{Correlations among simple acuity measures and cognition}

As was noted in the introduction and again in the discussion, it is argued that the nature of the sensory measures obtained is critical and that simple "clinical" measures of acuity alone, such as Snellen charts for visual acuity and puretone audiograms for hearing acuity, are insufficient. To assess this, since such simple measures of visual and auditory acuity were also obtained from each subject in this study, the associations among these two measures of acuity, age, and CogProc_Global (from prior PC analysis) were examined. Table 7 presents the Pearson $r$ correlation coefficients among this set of four measures. All correlations are weak to moderate in strength and statistically significant $(p<.001)$. Age is seen to be positively correlated to both clinical measures of sensory acuity, such that older individuals have greater loss of acuity in both senses, whereas the association with age and cognition is negative, as was expected. Moreover, there are some weak negative associations between sensory acuity in either hearing or vision and cognitive processing-again, as has been observed many times with such measures. Recall from the preceding analyses that controlling for sensory processing differences
Table 7 Summary of Pearson $r$ correlations observed between the CogProc_Global factor scores, age, and clinical measures of sensory acuity for hearing (high-frequency pure-tone average) and vision (Snellen chart, expressed as LogMAR values)

\begin{tabular}{llcc}
\hline & CogProc_Global & Visual acuity & Hearing loss \\
\hline Age & $-.55^{*}$ & $.37^{*}$ & $.66^{*}$ \\
CogProc_Global & & $-.32^{*}$ & $-.42^{*}$ \\
Visual acuity & & & $.30^{*}$ \\
\hline
\end{tabular}

Note. Asterisks mark correlations that are statistically significant, $p<.001$.

eliminated the association between age and cognitive processing. Here, however, when partial correlations were computed between age and CogProc_Global, controlling for hearing acuity, visual acuity, and both, the resulting partial correlation between age and cognitive processing remained moderate and statistically significant, with values of $r=-.41,-.52$, and -.39 , respectively. Thus, individual differences in simple clinical measures of acuity do not appear to mediate the observed association between age and cognitive processing. Likewise, computing partial correlations between age and each of the acuity measures, while controlling for cognitive processing, resulted in little attenuation of the correlations provided in Table 7. Specifically, the correlations between age and visual acuity and between age and hearing loss were .24 and .57 , respectively, when cognitive processing was controlled for. Finally, the weak negative, but statistically significant, correlations between cognitive processing and each of the sensory acuity measures (Table 7) decreased to nonsignificant near-zero correlations $(r=-.08$ for hearing loss and $r=-.14$ for visual acuity) when age was controlled for. From this overall pattern of correlations (Table 7) and partial correlations, age is associated with declining sensory acuity and declining cognitive processing, but this does not appear to be driven by an association between sensory acuity and cognition. In fact, when age was controlled for, no such association between sensory acuity and cognitive processing was found in this study when relying on simple clinical measures of sensory acuity.

At first glance, these correlations may appear to be at odds with the recent findings of Lin and colleagues (Lin, 2011; Lin, Ferrucci, et al., 2011; Lin, Metter, et al., 2011). In these epidemiological studies, links have been established between simple measures of auditory acuity (pure-tone audiometry) and various measures of cognitive function, including incident dementia. Because of the different types of cognitive measures and statistical approaches, it is difficult to compare our results with the results from these epidemiological studies. However, in Lin, a scatterplot and Pearson $r$ correlation coefficient for the association between average 
audiometric hearing loss and the lone cognitive measure in that study, the Digit-Symbol Substitution Test, are provided. The correlation was weak $(r=-.18)$ but highly significant $(p<.001)$, given the large sample size. On the basis of visual inspection of the scatterplots and the statistical analyses in Lin, Ferrucci, et al., it appears that the Pearson $r$ correlations between hearing loss and several different cognitive measures is similar to that reported by Lin - that is, weak but statistically significant. Thus, the weak to moderate correlations observed in the present study between simple measures of auditory acuity and cognitive function appear to be generally consistent with the weak correlations reported by Lin and colleagues.

With regard to the four hypotheses noted by Schneider and Pichora-Fuller (2000) and reviewed briefly in the introduction regarding various alternatives for the interaction of sensory and cognitive processing, the present results help clarify the kinds of information degradation that are consistent with each model, as described below. The data from this study suggest that there is a link between psychophysical measures of sensory processing and cognitive function in adults, regardless of the age of the adult. The correlations establish the magnitude of this association but do not indicate whether better sensory processing leads to better cognitive function (the sensory deprivation and information degradation hypotheses) or vice versa (the cognitive-loadon-perception hypothesis). Since SensProc_Global essentially represents the shared variance across many of the eight sensory-processing components (Table 4), including simple measures of detection threshold (audtacTHR) and gap detection (audGD), it would seem less likely that performance on these simple detection measures would be determined by cognitive function and more likely that the association would be in the opposite direction - that is, sensory processing mediating cognition. An association in this direction would be more consistent with the sensory deprivation and information degradation hypotheses. As has been noted, the primary difference between these two hypotheses is in the time course over which the negative peripheral effects impact cognitive performance, with deprivation assumed to be a chronic condition and information degradation more acute, impacting performance while measured, but not necessarily enduring. It should be noted that considerable care was taken in this study to minimize the impact of peripheral acuity deficits on performance. For example, the vowel stimuli used in phases 2 and 3 of this study were presented at high levels ( $83 \mathrm{~dB}$ SPL) and after lowpass filtering the stimuli at $1800 \mathrm{~Hz}$ to minimize any impact of peripheral high-frequency hearing loss on performance. In similar fashion, the contrast of the visual stimuli was adjusted for the older adults to enhance identification of the letters used in the phase 2 and 3 temporal order and temporal-masking studies. For all senses, hearing, vision, and touch, moreover, the screening protocol required at least $80 \%$ correct identification of the stimuli used in phases 2 and 3 prior to participation. Finally, when the WAIS-III was administered, an assistive-listening device was made available to those who needed it to make sure that performance on the auditory-based cognitive tasks was not impaired due to inaudibility, and visually based cognitive tasks were performed with the subject's corrective lenses. Thus, it is believed that acute effects of peripheral acuity deficits on performance for the sensory temporal-processing or cognitive-processing measures were minimized in this study, thereby making the influence of information degradation less relevant. However, if the information degraded is not related to sensory acuity per se but is, instead, related to the temporal encoding of sensory information, it remains possible that this form of information degradation could underlie the link between sensory processing and cognition observed in this study.

If information degradation is a more viable explanation than sensory deprivation, given that the cognitive measures obtained in this study via the WAIS-III were primarily visual, one might expect a stronger association between visual sensory processing and global cognitive function. To examine this, Pearson $r$ correlations were calculated between each of the eight psychophysical sensoryprocessing PCs (Table 3) and CogProc_Global. Although all eight correlations were significant $(p<.001)$ and weak to moderate in strength $(-.19<r<-.68)$, the correlations for visual measures were generally weaker $(r=-.34,-.37$; visFF and visTOTM, respectively) than those for hearing $(r=-.56,-.53$; audGD and audTOTM, respectively), touch $(r=-.19,-.68$; tacGD and tacTOTM, respectively), or hearing and touch combined $(r=-.48$, audtacTHR). (The remaining correlation, between audtacvisTOloc and CogProc_Global, was -.47.) Thus, although significant associations between visual sensory processing and performance on the predominantly visual WAIS-III were observed, even stronger associations were observed between auditory and tactile sensory processing and cognitive function. This would tend to argue against the information degradation hypothesis and also serves to emphasize the importance of global sensory-processing performance to global cognitive function. That is, no one sense or task seems to predominate among the set of sensory-processing measures as a predictor of global cognitive processing.

Again, it is important to emphasize that this link between sensory processing and cognition was observed even when age was controlled statistically via partial correlations. This would argue against the common-cause hypothesis for agerelated changes in cognition. That is, both sensory processing and cognition do not need to be impacted concurrently by the same common underlying mechanism with advancing age. However, there may be a role for a common cause 
that impacts multiple sensory systems concurrently (SensProc_Global), and perhaps in multiple ways (impacting threshold sensitivity and temporal processing), with advancing age, which, in turn, impacts cognition. Aging appears to operate at the sensory common-cause level, such that older individuals tend to have poorer sensory acuity and temporal processing in multiple senses than do young adults and this, in turn, is associated with poorer cognitive function in these same individuals. This finding represents one of the most important outcomes of this study.

Ideally, longitudinal study of sensory and cognitive processes over the adult lifespan will be conducted in the future to further investigate possible sequential effects and further delineate among the candidate hypotheses. Toward this end, the results of the present study can be used to establish the number and type of sensory measures needed to obtain a fairly comprehensive battery of reliable sensory-processing measures, several of which are related to aging and general cognitive function. On the basis of the PC analysis of the 40 psychophysical measures included in this comprehensive study, for example, no more than 8 such measures, 1 for each PC, would be needed in the future to provide a fairly comprehensive picture of "sensory processing." Furthermore, on the basis of an evaluation of the reliability of the measures obtained in this study, fewer than the 200-250 trials per threshold estimate would be required for reliable estimates of performance in the future.

\section{Conclusion}

In conclusion, we reexamined the relationship between age, cognition, and sensory processing across the adult lifespan. This was accomplished using reliable, psychophysically rigorous measures of sensory acuity and temporal processing in hearing, vision, and touch. Our data clearly showed a decline in nearly all 40 sensory measures with advancing age, as was expected, commensurate with an overall strong correlation between age and SensProc_Global $(r=.75)$. Of course, responses in several of the sensory tasks involved cognitive processes, so when the CogProc_Global contribution was partialed out, the relationship between age and SensProc_Global decreased, although it was still strong at $r_{\mathrm{p}}=.61$. A very different picture arose when the cognitive measures were examined. Whereas, as was expected, performance on the individual and global cognitive measures declined with age, when the SensProc Global contribution was partialed out, there was no observable relationship between age and CogProc_Global $\left(r_{\mathrm{p}}=-.05\right)$. An equally surprising result was that the partial correlation between SensProc_Global and CogProc_Global of $r_{\mathrm{p}}=-.53$ indicated that, at any age, there was a reasonably strong relationship between performance on these sensory and cognitive tasks. Similar associations were also confirmed using SEM and different approaches to data transformation and the treatment of missing data. This suggests that the conclusions noted above are robust and not confined to the particular choice of analysis model. Just as importantly, such associations, were not confirmed when "sensory processing" was represented by simple "clinical" measures of visual and auditory acuity (as in most prior studies). Although causality is not determined from correlations, directionality may safely be inferred from the nature of the tasks involved. All of our sensory and cognitive tasks involved the presentation of stimuli. Cognition could be engaged only after some aspects of the stimuli were processed by the sensory system stimulated, recalling that the psychophysical procedures used in the sensory tasks minimized bias. Logically, then, directionality is implied, with sensory processing largely preceding cognition in these laboratory tasks. Thus, an important and unexpected conclusion can be drawn from this study. Age negatively and primarily influenced the abilities to extract sensory information from the environment, with observed cognitive deficits mediated by such sensory deficits.

Acknowledgments The authors express their deep gratitude to the many subjects who devoted many hours to this study. We thank Dana Kinney for her assistance with project management and data collection, Christopher Clark, Roger Rhodes, Shamim Razawi, and Dan Fogerty for their assistance with data collection, and Martin Rickert for assistance with data analysis. This study was supported, in part, by NIA research grant R01-AG022334.

Open Access This article is distributed under the terms of the Creative Commons Attribution License which permits any use, distribution, and reproduction in any medium, provided the original author(s) and the source are credited.

\section{References}

Amberson, J. I., Atkeson, B. M., Pollack, R. H., \& Malatesta, V. J. (1979). Age difference in dark-interval threshold across the lifespan. Experimental Aging Research, 5, 423-433.

American National Standards Institute. (2004). Specification for audiometers, ANSI S3.6-2004. Melville, NY: ANSI.

Bergner, M., Bobbitt, R. A., Carter, W. B., \& Gilson, B. S. (1981). The Sickness Impact Profile: Development and final revision of a health status measure. Medical Care, 19, 787-805.

Busey, T., Craig, J., Clark, C., \& Humes, L. E. (2010). Age-related changes in visual temporal order judgment performance: Relation to sensory and cognitive capacities. Vision Research, 50, 1628-1640.

Cholewiak, R. W., \& Craig, J. C. (1984). Vibrotactile pattern recognition and discrimination at several body sites. Perception \& Psychophysics, 35, 503-514.

Clark, W. C., \& Mehl, L. (1971). Thermal pain: A sensory decision theory analysis of the effect of age and sex on d', various response criteria, and $50 \%$ pain threshold. Journal of Abnormal Psychology, 78, 202-212.

Craig, J. C., Rhodes, R., Busey, T. A., Kewley-Port, D., \& Humes, L. E. (2010). Aging and tactile temporal order. Attention, Perception, \& Psychophysics, 72, 226-235. 
Evans, A. S. (1978). Causation and disease: A chronological journey. American Journal of Epidemiology, 108(4), 248-255.

Fogerty, D., Humes, L. E., \& Kewley-Port, D. (2010). Auditory temporal-order processing of vowel sequences by young and elderly listeners. Journal of the Acoustical Society of America, 127, 2509-2520.

Folstein, M. F., Folstein, S. E., \& McHugh, P. R. (1975). Mini-Mental State: A practical method for grading the cognitive state of patients for the clinician. Journal of Psychiatric Research, 12, 189-198.

Fozard, J. L. (1990). Vision and hearing in aging. In J. E. Birren \& K. W. Schaie (Eds.), Handbook of the psychology of aging (3rd ed., pp. 150-170). New York: Academic Press.

Fraisse, P. (1984). Perception and estimation of time. Annual Reviews in Psychology, 35, 1-36.

Gatehouse, S., \& Davis, A. (1992). Clinical pure-tone versus threeinterval forced-choice thresholds: Effects of hearing level and age. International Journal of Audiology, 31, 31-44.

Gordon-Salant, S. (1986). Effects of aging on response criteria in speech-recognition tasks. Journal of Speech and Hearing Research, 29, 155-162.

Gordon-Salant, S., \& Fitzgibbons, P. J. (1999). Profile of auditory temporal processing in older listeners. Journal of Speech and Hearing Research, 42, 300-311.

Gorsuch, R.L. (1983). Factor analysis (2nd ed.). Lawrence Erlbaum Associates.

Green, D. M., \& Swets, J. A. (1966). Signal detection theory and psychophysics. New York: Wiley.

Humes, L. E., Busey, T. A., Craig, J. C., \& Kewley-Port, D. (2009). The effects of age on sensory thresholds and temporal gap detection in hearing, vision and touch. Attention, Perception, \& Psychophysics, 71, 860-871.

Humes, L. E., Kewley-Port, D., Fogerty, D., \& Kinney, D. (2010). Measures of hearing threshold and temporal processing across the adult lifespan. Hearing Research, 264, 30-40.

James, W. (1890). The principles of psychology. New York: Holt.

Kruskal, J. B., \& Shepard, R. N. (1974). A nonmetric variety of linear factor analysis. Psychometrika, 39(2), 123-157.

Levitt, H. (1971). Transformed up-down method in psychoacoustics. Journal of the Acoustical Society of America, 49, 467-477.

Lin, F. R. (2011). Hearing loss and cognition among older adults in the United States. Journal of Gerontology: Medical Sciences, 66A, 1131-1136.

Lin, F. R., Ferrucci, L., Metter, E. J., An, Y., Zonderman, A. B., \& Resnick, S. M. (2011a). Hearing loss and cognition in the Baltimore Longitudinal Study of Aging. Neuropsychology, 25, 763-770.

Lin, F. R., Metter, E. J., O’Brien, R. J., Resnick, S. M., Zonderman, A. B., \& Ferrucci, L. (2011b). Hearing loss and incident dementia. Archives of Neurology, 68, 214-220.

Lindenberger, U., \& Baltes, P. B. (1994). Sensory functioning and intelligence in old age: A strong connection. Psychology and Aging, 9, 339-355.

Little, R. J. A. (1988). Missing data adjustments in large surveys (with discussion). Journal of Business Economics and Statistics, 6 , 287-301.
Marshall, L. (1991). Decision criteria for pure-tone detection used by two age groups of normal-hearing and hearing-impaired listeners. Journal of Gerontology, 46, P67-P70.

Moore, B. C. J., Peters, R. W., \& Glasberg, B. R. (1992). Detection of temporal gaps in sinusoids by elderly subjects with and without hearing loss. Journal of the Acoustical Society of America, 92, 1923-1932.

Potash, M., \& Jones, B. (1977). Aging and decision criteria for the detection of tones in noise. Journal of Gerontology, 32, 436-440.

Ram, N., Lindenberger, U., \& Blanchard-Fields, F. (2009). Introduction to the special section on intraindividual variability and aging. Psychology and Aging, 24, 775-777.

Ratcliff, R., Thapar, A., \& McKoon, G. (2007). Application of the diffusion model to two-choices tasks for adults 75-90 years old. Psychology and Aging, 22, 56-66.

Rees, J. N., \& Botwinick, J. (1980). Detection and decision factors in auditory behavior of the elderly. Journal of Gerontology, 35, 133-136.

Salthouse, T. A. (2010). Major issues in cognitive aging. New York: Oxford University Press.

Schacht, J., \& Hawkins, J. E., Jr. (2005). Sketches of Otohistory. Part 9: Presby(a)cusis. Audiology and Neurotology, 10, 243-247.

Schaie, K. W. (1983). Longitudinal studies of adult psychological development. New York: Guilford Press.

Schneider, B. A., \& Pichora-Fuller, M. K. (2000). Implications of perceptual processing for cognitive aging research. In F. I. M. Craik \& T. A. Salthouse (Eds.), The handbook of aging and cognition (2nd ed.). New York: Lawrence Erlbaum Associates.

Schneider, B. S., Pichora-Fuller, M. K., Kowalchuk, D., \& Lamb, M. (1994). Gap detection and the precedence effect in young and old adults. Journal of the Acoustical Society of America, 95, 980-991.

Stevens, J. C., Cruz, L. A., Marks, L. E., \& Lakatos, S. (1998). A multimodal assessment of sensory thresholds in aging. Journal of Gerontology, 53B, P263-P272.

Strouse, A., Ashmead, D. H., Ohde, R. N., \& Granthan, D. W. (1998). Temporal processing in the aging auditory system. Journal of the Acoustical Society of America, 104, 2385-2399.

van Buuren, S., \& Groothuis-Oudshoorn, K. (2011). MICE: Mulitivariate Imputation by Chained Equations in R. Journal of Statistical Software, 45(3), 1-61.

van Buuren. S. \& Oudshoorn, C.G.M. (2000). Multivariate Imputation by Chained Equations: MICE V1.0 User's manual. TNO Report PG/VGZ/00.038. Leiden: TNO Preventie en Gezondheid.

Van Doren, C. L., Gescheider, G. A., \& Verrillo, R. T. (1990). Vibrotactile temporal gap detection as a function of age. Journal of the Acoustical Society of America, 87, 2201-2206.

Watson, C. S., Turpenoff, C. M., Kelly, W. J., \& Botwinick, J. (1979). Age differences in resolving power and decision strategies in a weight discrimination task. Journal of Gerontology, 34, 547-552.

Wechsler, D. (1997). Wechsler adult intelligence scale, 3rd Edition (WAIS-III). San Antonio, TX: The Psychological Corporation.

Woodward, J. A., \& Overall, J. E. (1976). Factor analysis of rankordered data: An old approach revisited. Psychological Bulletin, 83, 864-867. 